\title{
Gestão da rede de atenção psicossocial: uma revisão integrativa
}

\author{
Management of the psychosocial care network: an integrative review \\ Gestión de la red de atención psicosocial: una revisión integradora
}

Recebido: 17/08/2021 | Revisado: 22/08/2021 | Aceito: 25/08/2021 | Publicado: 28/08/2021

\author{
Arcanjo Sousa \\ ORCID: https://orcid.org/0000-0002-4300-6504 \\ Universidade Estadual do Ceará, Brasil \\ E-mail: arcanjosousa14@gmail.com \\ Maria Salete Bessa Jorge \\ ORCID: https://orcid.org/0000-0001-6461-3015 \\ Universidade Estadual do Ceará, Brasil \\ E-mail: maria.salete.jorge@gmail.com \\ Dina Mara Formiga da Silva \\ ORCID: https://orcid.org/0000-0002-5387-7194 \\ Universidade Estadual do Ceará, Brasil \\ E-mail: dinamara_silva@ hotmail.com \\ Débora Brenda Carneiro de Souza \\ ORCID: https://orcid.org/0000-0002-8503-4052 \\ Universidade Estadual do Ceará, Brasil \\ E-mail: deborabrenda@outlook.com \\ Cristiane Sales Leitão \\ ORCID: https://orcid.org/0000-0002-8412-0629 \\ Assembleia Legislativa do Ceará, Brasil \\ E-mail: cristianesalesleitao@gmail.com \\ Paulo Barroso \\ ORCID: https://orcid.org/0000-0002-4902-1940 \\ Assembleia Legislativa do Ceará, Brasil \\ E-mail:psi.pbarrodo@gmail.com \\ Lídia Lourinho \\ ORCID: https://orcid.org/0000-0002-5883-9007 \\ Assembleia Legislativa do Ceará, Brasil \\ E-mail: lidialourinho@ hotmail.com
}

\begin{abstract}
Resumo
Objetivo: analisar na literatura, como ocorre a gestão da Rede de Atenção Psicossocial e os participantes deste processo, levando em consideração a participação social através do Conselho Nacional de Saúde. Método: Revisão Integrativa, que tem como base as seis fases estabelecidas por Mendes, Silveira e Galvão, sendo utilizada a estratégia PCC para a formulação da pergunta norteadora. Resultados: Foram escolhidos um total de 10 artigos Discussão: Através da análise dos artigos por meio de leitura intensiva, as seguintes categorias foram formuladas: Processo da gestão da Rede de Atenção Psicossocial e Participantes do processo de gestão. Conclusão: Os gestores, as equipes multiprofissionais e os usuários necessitam trabalhar em conjunto para gerir a rede a fim de que haja o estabelecimento de metas e planejamentos que supram as necessidades dela.
\end{abstract}

Palavras-chave: Equipe; Gestão; Rede de Atenção Psicossocial.

\section{Abstract}

Objective: to analyze in the literature, how the psychosocial care network is management and the participants of this process, taking into account social participation through the National Health Council. Method: Integrative Review, which is based on the six phases established by Mendes, Silveira and Galvão, using the PCC strategy for the formulation of the guide question. Results: A total of 10 articles discussion were chosen: Through the analysis of the articles through intensive reading, the following categories were formulated: Process of management of the Psychosocial Care Network and Participants of the management process. Conclusion: Managers, multidisciplinary teams and users need to work together to manage the network so that there is the establishment of goals and planning that meet its needs.

Keywords: Team; Management; Psychosocial Care Network.

\section{Resumen}

Objetivo: analizar en la literatura, cómo es la gestión de la red de atención psicosocial y los participantes de este proceso, teniendo en cuenta la participación social a través del Consejo Nacional de Salud. Metodo: Revisión 
Integrativa, que se basa en las seis fases establecidas por Mendes, Silveira y Galvão, utilizando la estrategia del PCC para la formulación de la pregunta guía. Resultados: Se eligieron un total de 10 artículos de discusión: A través del análisis de los artículos a través de la lectura intensiva, se formularon las siguientes categorías: Proceso de gestión de la Red de Atención Psicosocial y Participantes del proceso de gestión. Conclusión: Los gestores, equipos multidisciplinares y usuarios necesitan trabajar juntos para gestionar la red de manera que se establezcan objetivos y planificación que satisfagan sus necesidades.

Palabras clave: Equipo; Administración; Red de Atención Psicosocial.

\section{Introduçãa}

A Saúde Mental é definida pela Organização Mundial de Saúde (OMS) como um estado de bem-estar, onde o indivíduo consegue usar suas próprias habilidades, recuperar-se do estresse rotineiro, ser produtivo e contribuir com a sua comunidade, para tanto, tal feito é ofertado no Sistema Único de Saúde (SUS) em organizações de níveis de complexidade. (Ministério da Saúde, 2019).

A Rede de Atenção Psicossocial (RAPS), foi formada com o objetivo de criar, ampliar e articular pontos de atenção à saúde para usuários que tenham sofrimento ou transtorno mental e com necessidades devido ao uso de crack, álcool e outras drogas no âmbito do Sistema Único de Saúde (Ministério da Saúde, 2011).

Instituída dentro do campo da Saúde Mental, no ano de 2011, pela Portaria 3.088, a RAPS veio com o objetivo de melhorar a atenção à saúde nessa área, tornando-a mais integrada, contando com o apoio de diversos pontos da rede (Ministério da Saúde, 2011; Cardoso et al. 2017).

É composta por diversos pontos de acesso para os usuários, gerando uma diversidade de formas de atendimento para garantir a qualidade e eficiência do mesmo, mas para que isso ocorra é necessário que esses pontos estejam em harmonia entre si de forma integral, transmitindo informações de usuários entre os pontos de atendimento que precisam ir (Quinderé; Jorge \& Franco, 2014).

A RAPS precisa de uma forma eficiente de gestão, onde consiga trabalhar de forma integral com os usuários a fim de suprir suas necessidades. Um dos órgãos que existe para auxiliar nesse quesito é o Conselho Nacional de Saúde (CNS), que tem como missão a deliberação, fiscalização, acompanhamento e monitoramento de políticas públicas de saúde (Ibes, 2016).

O CNS é um órgão vinculado ao Ministério da Saúde, que tem em sua composição representantes de entidades e movimentos representativos de usuários, entidades que representam trabalhadores da área da saúde, governo e prestadores de serviços de saúde, dando, assim, importância a participação social em suas questões (Ibes, 2016; Ministério da Saúde, 2018).

Os participantes do CNS decidem entre si questões de suma importância para a saúde, como a aprovação do orçamento de saúde e o acompanhamento de sua execução orçamentária, aprovar o Plano Nacional de Saúde a cada quatro anos, dentre outras (Ibes, 2016; Ministério da Saúde, 2018).

A participação social dentro das questões de saúde, desde a promulgação da Constituição Federal de 1988, passou a ser valorizada, juntamente com a descentralização das ações e políticas de saúde e da integralidade da assistência, tornando-se de fundamental importância para que houvesse a formação de um modelo, de fato, público de saúde (Ministério da Saúde, 2018). Frente a isto, o presente estudo tem como objetivo analisar, na literatura, como ocorre a gestão da Rede de Atenção Psicossocial e os participantes deste processo, levando em consideração a participação social através do Conselho Nacional de Saúde.

\section{Metodologia}

Utilizou-se do método Revisão Integrativa (RI), que tem como base os seis estágios estabelecidos por Mendes; Silveira \& Galvão (2008), 1) definição do tema e elaboração da pergunta de pesquisa; 2) critérios de elegibilidade, inclusão e exclusão dos estudos; 3) levantamento dos estudos selecionados em formato de tabelas, considerando todas as características 
em comum; 4) categorização e análise crítica dos achados, identificando diferenças e conflitos; 5) interpretação dos resultados e 6) apresentação dos resultados, incluindo análise crítica dos achados e síntese da revisão.

Para a formulação da questão norteadora do estudo utilizamos a pergunta PCC, a estratégia PICO adaptada. Na adaptação, tal acrônimo significa P- população, C- conceito e C- contexto/desfecho. Assim, a estratégia foi formulada da seguinte maneira: P- profissionais; C- conselho de saúde mental na gestão; C- rede de atenção psicossocial (Peters et al. 2015).

Desta forma, estabeleceu-se, a seguinte questão: como ocorre a gestão da rede de atenção psicossocial, levando em consideração o nível de conhecimento dos profissionais da saúde de nível superior e os órgãos envolvidos nesse processo, ou seja, informações sobre como o mesmo ocorre, que possam ser encontradas na literatura Brasileira nos últimos cinco anos?

Após identificação dos Descritores em Ciências da Saúde (DeCS), uma estratégia de busca detalhada, e individual, foi aplicada em cada base de dados: Biblioteca Virtual em Saúde (BVS), Scielo. Sendo incluído todos os artigos publicados até junho de 2021, nos últimos cinco anos.

Utilizou-se três descritores extraídos do DeCS (Descritores em Ciências da Saúde) "profissionais de saúde", "administração de serviços de saúde", "serviços de saúde mental”, e um termo alternativo "gestão dos serviços de saúde". E para a busca complementar utilizou-se de literatura cinza, o Google Scholar, a fim de ampliação da busca por artigos sobre a temática.

Durante a estratégia de busca (Quadro1), foram elencados como critérios de inclusão: artigos completos disponíveis integralmente nas bases de dados elencadas nos idiomas português, inglês e espanhol, e selecionados artigos referentes aos últimos cinco anos, informações complementares utilizando-se de literatura cinza: Google Scholar; publicações que atendessem a pergunta norteadora. Foram excluídas publicações que preenchiam pelo menos um dos seguintes critérios: (1) revisões; (2) cartas ao editor, (3) opinião pessoal de autores; (4) resumo de encontros; (5) vídeos; (6) ausência de dados relacionados ao objeto e estudo.

Quadro 1 - Equações de buscas nas bases de dados.

$\begin{array}{ll}\text { ESTRATÉGIA DE BUSCA } \\ \text { BASE } & \text { (profissionais de saúde) AND (administração de serviços de saúde) AND (serviços de saúde mental) AND } \\ & \text { ( fulltext:("1" OR "1" OR "1" OR "1") AND db:("MEDLINE" AND "LILACS") AND } \\ & \text { type_of_study:("qualitative_research" OR "prognostic_studies" OR "risk_factors_studies" OR } \\ & \text { "prevalence_studies" OR "diagnostic_studies" OR "etiology_studies" OR "screening_studies" OR } \\ & \text { "guideline" OR "evaluation_studies" OR "observational_studies" OR "clinical_trials" OR } \\ & \text { "health_economic_evaluation" OR "case_reports" OR "incidence_studies" OR "overview" OR } \\ & \text { "policy_brief") AND la:("pt")) AND (year_cluster:[2017 TO 2021]), }\end{array}$

SCIELO (profissionais de saúde) AND (administração de serviços de saúde) OR (gestão dos serviços de saúde) AND (serviços de saúde mental) AND type_of_study:(“article" OR "case_report” OR "rapid_communication" OR "undefined" OR "editorial” OR "brief_report" OR "article_commentary" OR “letter” OR “press_release” OR “addendum”) AND la:(“pt”)) AND (year_cluster:[2017 TO 2021])

Fonte: Autores (2021).

A combinação dos termos foi selecionada e adaptada para cada base de dados (Quadro 1). O software Endnotweb foi selecionado para o manejo de todas as referências e a remoção dos artigos duplicados. Para a análise dos dados, foi utilizada a Análise de Conteúdo de Bardin (2011), estabelecendo assim categorias. 


\section{Resultados}

A busca resultou inicialmente em $(n=46)$ artigos, tendo a seguinte distribuição entre as publicações encontradas em cada base de dados: BVS $(n=29)$; Scielo $(n=17)$; Literatura cinza, com Google Scholar $(n=100)$, totalizando $(n=146)$ publicações. Em seguida, foram analisadas as publicações $(n=146)$, destas foram excluídas ( $n=126)$ por não se adequarem aos critérios de inclusão da pesquisa. Assim, restaram $(n=20)$ para serem analisadas, onde $(n=10)$ foram excluídas por se encaixarem em um dos critérios de exclusão.

Figura 1- Fluxograma da seleção das publicações para a revisão integrativa baseado no modelo PRISMA.
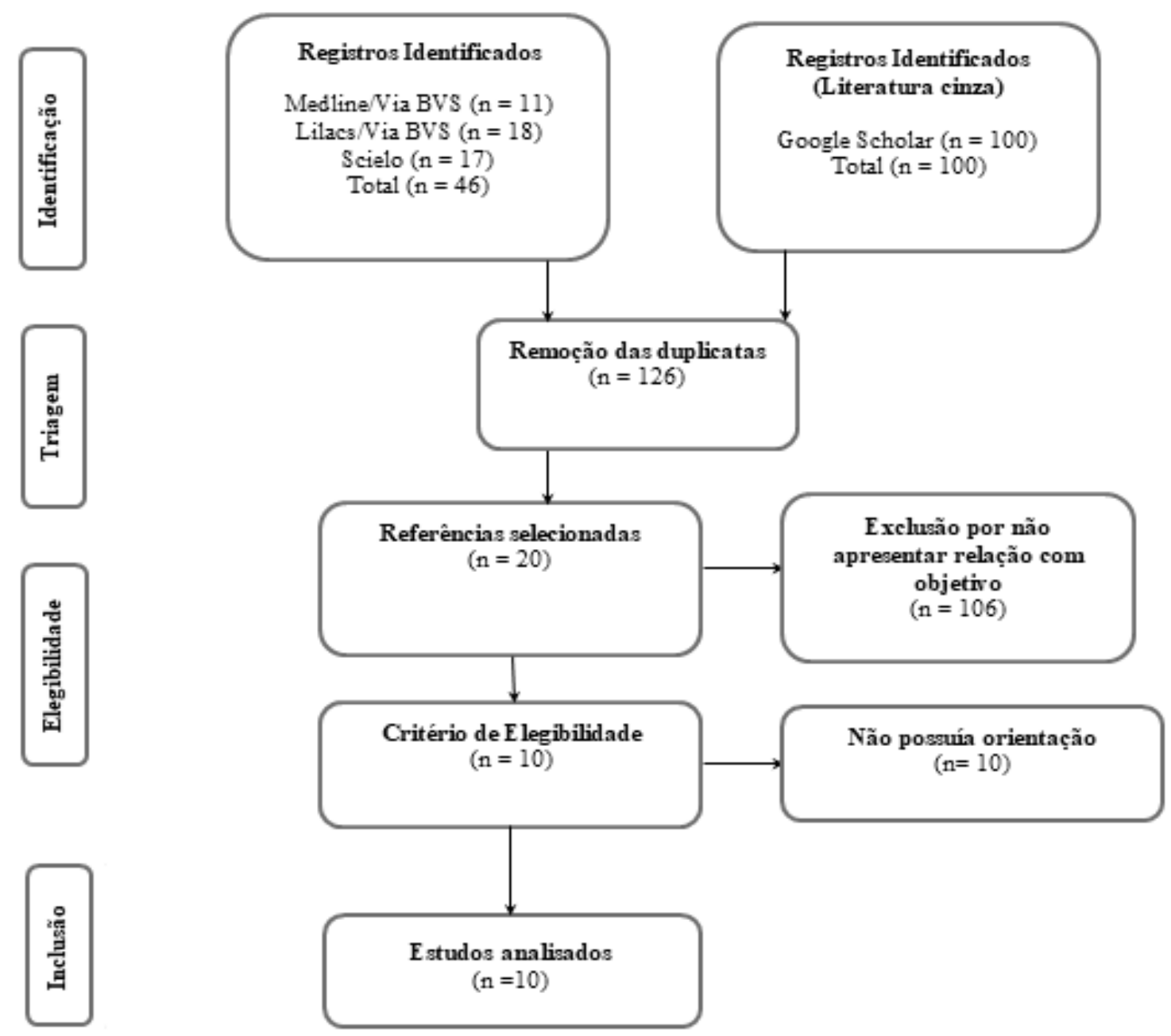

Fonte: Autores (2021).

As etapas deste processo foram descritas na forma de um fluxograma (Figura 1) adaptado do Reporting Items Systematic and Meta-Analyses (PRISMA) restando (n=10) para serem analisados e construída a matriz de síntese (Quadro 2). 
Quadro 2 - Caracterização dos estudos incluídos na Revisão Integrativa.

\begin{tabular}{|c|c|c|c|c|c|}
\hline Título & Autor & Idioma & Periódico & $\begin{array}{l}\text { Tipo de } \\
\text { publicação }\end{array}$ & Principais resultados \\
\hline $\begin{array}{l}\text { A tessitura dos encontros da Rede } \\
\text { de Atenção Psicossocial }\end{array}$ & $\begin{array}{l}\text { Leite, L. S.; } \\
\text { Rocha, K. B.; } \\
\text { Santos, L. M. }\end{array}$ & Português/Inglês & Trab. Educ. Saúde & $\begin{array}{l}\text { Estudo com } \\
\text { abordagem } \\
\text { qualitativa }\end{array}$ & $\begin{array}{l}\text { A composição do fórum, que conta com profissionais de } \\
\text { diversos serviços, demarca um processo que visa articular } \\
\text { diferentes pontos da Rede de Atenção Psicossocial, } \\
\text { promovendo interlocução entre os serviços e a } \\
\text { corresponsabilidade pela gestão-execução da atenção em saúde } \\
\text { no território. }\end{array}$ \\
\hline $\begin{array}{c}\text { A saúde mental e atenção } \\
\text { psicossocial: regionalização e } \\
\text { gestão do cuidado integral no SUS }\end{array}$ & $\begin{array}{l}\text { Dimenstein, M. } \\
\quad \text { el al. }\end{array}$ & $\begin{array}{l}\text { Português/Inglês/ } \\
\text { Espanhol }\end{array}$ & Salud \& Sociedad & $\begin{array}{l}\text { Estudo de } \\
\text { corte } \\
\text { transversal }\end{array}$ & $\begin{array}{l}\text { Foram identificadas fragilidades na articulação entre saúde } \\
\text { mental e atenção primária e } \\
\text { em relação à longitudinalidade e coordenação do cuidado, à } \\
\text { formação de vínculos, à orientação do trabalho para a } \\
\text { comunidade e famílias e à competência cultural das equipes. }\end{array}$ \\
\hline $\begin{array}{l}\text { Articulação da Rede de Atenção } \\
\text { Psicossocial e continuidade do } \\
\text { cuidado em território: } \\
\text { problematizando possíveis relações }\end{array}$ & $\begin{array}{l}\text { Lima, D. K. R. } \\
\text { R.; Guimarães, } \\
\text { J. }\end{array}$ & Português/Inglês & $\begin{array}{l}\text { Physis: Revista de } \\
\text { Saúde Coletiva }\end{array}$ & $\begin{array}{l}\text { Pesquisa de } \\
\text { campo com } \\
\text { abordagem } \\
\text { qualitativa }\end{array}$ & $\begin{array}{l}\text { Chama-se a atenção para a recursividade que se estabelece } \\
\text { entre o cuidado em território e a articulação da RAPS. }\end{array}$ \\
\hline $\begin{array}{l}\text { Avaliação de Serviços de Saúde } \\
\text { Mental Brasileiros: satisfação dos } \\
\text { usuários e fatores associados }\end{array}$ & $\begin{array}{l}\text { Silva, S. N.; } \\
\text { Lima, M. G.; } \\
\text { Ruas, C. M. }\end{array}$ & Português/Inglês. & $\begin{array}{l}\text { Ciência Saúde } \\
\text { Coletiva }\end{array}$ & $\begin{array}{l}\text { Estudo } \\
\text { transversal e } \\
\text { correlacional }\end{array}$ & $\begin{array}{l}\text { Condições físicas e conforto do serviço obtiveram os menores } \\
\text { escores na escala de avaliação de satisfação. }\end{array}$ \\
\hline $\begin{array}{l}\text { Desafios na construção de uma } \\
\text { Rede de Atenção Psicossocial no } \\
\text { município de Manaus (AM): } \\
\text { discursos e práticas de gestores e } \\
\text { profissionais. }\end{array}$ & Barbosa, T. L. & Português. & $\begin{array}{l}\text { Instituto de Medicina } \\
\text { Social }\end{array}$ & $\begin{array}{l}\text { Pesquisa de } \\
\text { campo } \\
\text { qualitativa }\end{array}$ & $\begin{array}{l}\text { A Rede de Atenção Psicossocial de Manaus foi caracterizada } \\
\text { como de baixa conectividade utilizando-se de estratégias de } \\
\text { integração pouco definidas, prevalecendo a rede de contatos } \\
\text { pessoais de seus profissionais e como insuficiente, em virtude } \\
\text { da baixa cobertura de serviços, os quais até então existentes, } \\
\text { foram viabilizados mediante intervenção judicial. }\end{array}$ \\
\hline
\end{tabular}


Research, Society and Development, v. 10, n. 11, e173101119513, 2021

(CC BY 4.0) | ISSN 2525-3409 | DOI: http://dx.doi.org/10.33448/rsd-v10i11.19513

Dispositivos e conexões da Rede de Atenção Psicossocial (RAPS)

de Porto Alegre - RS

Gestão do cuidado e interdisciplinaridade: desafios do cotidiano da atenção psicossocial

Meios de trabalho do enfermeiro na articulação da rede de atenção psicossocial

Os desafios da organização em rede na atenção psicossocial especializada: o caso do Recife
Percepção da equipe multidisciplinar sobre a estrutura dos serviços de saúde mental: estudo fenomenológico

$\begin{array}{lccc}\text { Zanardo, G. L. } & \text { Português/Inglês/ } & \text { Estudos } & \text { Pesquisa } \\ \text { P.; Bianchessi, } & \text { Espanhol } & \text { Interdisciplinares em } & \text { qualitativa. } \\ \text { D. L. C.; Rocha, } & & \text { Psicologia } & \end{array}$

K. B.

Pessoa, K. L. V. Português/Inglês/ et al. Espanhol

Rev. Salud Pública

Pesquisa qualitativa

Braga, F. S. et Português/Inglês Rev. Gaúcha Enferm. al.

Silva, A. P. et

Português

al.

Schran, L. S. et al.

Português/Inglês

Rev. Gaúcha Enferm

Estudo qualitativo exploratóriodescritivo

Estudo qualitativo

Estudo exploratório
A partir do exposto, considera-se que as equipes aderem ao movimento de mudança da lógica de assistência e esforçam-se em estreitar a comunicação entre si, embora existam práticas ainda incipientes.

Do cotidiano da atenção psicossocial emergiram subjetividades relacionadas à precarização do trabalho em saúde determinado pela infraestrutura física deficiente, quantidade e qualidade de material insatisfatórios, trazendo desalentos para o exercício das práticas e do cuidado com qualidade

análise resultou em duas categorias temáticas: Gestão do Cuidado e Práticas de Cuidado. A primeira é composta por dois meios de trabalho: coordenação de equipe e reuniões com os demais componentes do território. A segunda, envolve três meios de trabalho: contatos telefônicos com serviços da RAPS; encaminhamentos do usuário na RAPS e apoio matricial.

Apesar do histórico de alinhamento com a Reforma Psiquiátrica Brasileira e dos avanços nos processos de desinstitucionalização, a cobertura dos Centros de Atenção Psicossocial/tipo III é insuficiente e há fragilidades de comunicação entre os componentes da rede. Além disso, os ambulatórios de saúde mental atuam em complementaridade ao modelo manicomial.

A partir da análise foram identificadas as seguintes categorias: a estrutura organizacional da rede de atenção à saúde mental; ações de cuidado no contexto da estrutura organizacional dos serviços de saúde mental e; expectativas em relação à rede de atenção à saúde mental.

Fonte: Autores (2021). 


\section{Discussões}

Conforme o tempo passa os transtornos mentais acometem cada vez mais a população do país, aumentando os números de quem os têm. A Organização Mundial da Saúde (OMS) informa que o Brasil é o segundo maior país com número de depressivos (cerca de 5,8\%) e que tem a maior prevalência de ansiedade no mundo (9,3\%), onde estes são os maiores transtornos presentes no país (Hurso, 2020).

Para trabalhar a forma de condução da grande demanda de transtornos mentais, vem a Rede de Atenção Psicossocial (RAPS), com sua diversidade de pontos e trabalho integral (Ministério da Saúde, 2011). A RAPS é considerada uma ação prioritária da Política Nacional de Saúde Mental (PNSM), e tem como objetivo prestar um atendimento integral e humanizado para os indivíduos que estão em seu foco de atenção, com um modelo de atenção que seja aberto, ou seja, garanta a circulação das pessoas com problemas mentais pelos serviços, comunidade e cidade, um serviço de portas abertas em todos os seus pontos (Nóbrega; Silva \& Sena, 2016).

Desenvolvida para ser uma estratégia de enfrentamento das vulnerabilidades, agravos ou doenças que atingem as populações em suas regiões de vivência, sendo embasada na lógica de que muitos dos fatores e elementos geradores de transtorno mental são ligados a fatores do meio no qual estão inseridos ou são, propriamente, esses fatores (Nóbrega; Silva \& Sena, 2016).

Para se organizar de forma eficiente a RAPS tem sua divisão composta por pontos, cada um deles vêm se remeter a área e aos níveis de atenção, dos quais são: I - atenção básica em saúde; II - atenção psicossocial especializada; III - atenção de urgência e emergência; IV - atenção residencial de caráter transitório; V - atenção hospitalar; VI - estratégias de desinstitucionalização; e VII - reabilitação psicossocial (Ministério da Saúde, 2011).

Em 1990, através da lei 8.142, os conselhos e as conferências de saúde foram instituídos, como instrumentos de controle social, onde, através deles, deve haver participação de diversos segmentos existentes na sociedade, ao lado do governo, onde devem acompanhar e definir as políticas públicas de saúde (Ministério da Saúde, 2018).

O Conselho Nacional de Saúde (CNS) foi criado em 1937, com a lei 378, onde seu objetivo era assessorar o então Ministério da Educação e Saúde na época juntamente com o Conselho Nacional da Educação. No ano de 1990, com o decreto 99.438, o novo CNS foi criado, tendo sua base nos princípios e diretrizes do Sistema Único de Saúde (SUS) (Ministério da Saúde, 2016).

O CNS tem caráter deliberativo, onde, com isso, pode analisar e deliberar sobre assuntos de saúde pública, com resoluções, as quais o Ministério da Saúde deve adotar (Ibes, 2016; Ministério da Saúde, 2018).

Com o objetivo de deixar suas ações mais organizadas e integradas, o CNS criou diversas comissões, onde estas são responsáveis, cada uma, por uma área da saúde específica a qual foi designada, dentre elas está a Comissão Intersetorial de Saúde Mental (CISM) (Ministério da Saúde, 2019).

Instituída em 1999, a CISM veio com o objetivo de acompanhar a PNSM, onde fornece subsídios ao CNS para garantir o fortalecimento da RAPS. Suas ações tentam garantir a continuidade do Modelo de Atenção em Saúde Mental e da Reforma Psiquiátrica, que têm como base a desinstitucionalização dos usuários (Ministério da Saúde, 2018; Ministério da Saúde, 2019).

A comissão veio para acompanhar a implementação da Lei 10.216/2001, muito conhecida como a Lei da Reforma Psiquiátrica, e tem em sua composição diversos membros, dentre eles membros que representam a população, trazendo assim o caráter de importância da participação social que o CNS apresenta em seu modelo de funcionamento (Ministério da Saúde, 2020). 
A RAPS tem uma diversidade de atendimentos e pontos a serem geridos, para isso é necessário a existência do CNS que institui suas comissões, dentre elas, a CISM, que conta com a participação de diversos membros, como gestores, profissionais da saúde, usuários, dentre outros. A composição da comissão se torna de suma importância, levando em consideração seus membros usuários que fazem parte da população e são o alvo dos serviços, pois, com eles e suas opiniões, poderão criar estratégias de atendimento, tornando assim a atenção da rede o mais integral possível (Ministério da Saúde, 2020).

Após leitura e análise dos estudos encontrados nas bases de dados e na literatura cinza, seus dados foram reagrupados, permitindo, assim, a formulação das seguintes categorias: Processo da gestão da Rede de Atenção Psicossocial e Participantes do processo de gestão.

\subsection{Processo da gestão da Rede de Atenção Psicossocial}

A Rede de Atenção Psicossocial (RAPS) reflete um processo de desconstrução e reconstrução do modelo de atenção psicossocial, onde trabalha no contexto desafiador de desmonte do modelo manicomial historicamente instalado no sistema de saúde, com o propósito de prestar assistência de forma integral (Silva et al. 2021).

O cuidado em saúde mental não se baseia somente em um saber técnico de dimensão procedimental, ele engloba dimensões afetivas, éticas, ideológicas e políticas, onde possa ser expressa a singularidade do usuário, a fim de acolhê-lo do melhor jeito (Pessoa et al. 2018).

Para uma gestão eficiente da rede, primeiramente é necessário que haja um vínculo e uma conversação entre os dispositivos da mesma, pois ao conversarem entre si, passando informações e solucionando casos os dispositivos geram, verdadeiramente, um cuidado singular ao indivíduo, pois, assim, há a possibilidade de maior resolutividade das demandas apresentadas (Leite; Rocha \& Santos, 2017).

Sempre há encontros entre os dispositivos da rede, pois quando o usuário a adentra ele está sujeito a precisar de diversos tipos de atendimento que ela pode oferecer, desde a atenção primária até a especializada, o que possibilita o mesmo de ajudar na visão de gestão da rede. Quando há esse encontro e uma conversação da rede é possível que os profissionais atuantes nela possam se organizar melhor, fazendo com que vejam falhas ou novas necessidades, possibilitando a oportunidade da rede de se reinventar e gerir seus dispositivos de forma que possam atender às novas demandas, ou seja, gestores e profissionais têm uma visão melhor para manejar o funcionamento da rede e seus dispositivos (Dimenstein et al. 2018).

A rede conta com o apoio de vários dispositivos que auxiliam na formação e na funcionalidade dela, onde estão presentes o apoio matricial, ou matriciamento, que dá a oportunidade das equipes interagirem em apoio mútuo, as reuniões, onde podem ser discutidas questões da organização e gestão dos serviços, o contato imediato, onde há um trabalho e articulação por um contato mais direto e informal, a identificação da demanda, que permite o planejamento dos projetos terapêuticos e das intervenções, e a busca ativa, possibilitando o acompanhamento contínuo dos usuários de seu território (Leite; Rocha \& Santos, 2017; Zanardo; Bianchessi \& Rocha, 2018).

Ao utilizar os dispositivos auxiliadores e articular suas unidades, os responsáveis pela gestão da Rede de Atenção Psicossocial têm a possibilidade de realizar o rearranjo de suas estratégias, podendo organizar-se a fim de atender da melhor forma as necessidades apresentadas pelos usuários (Braga et al. 2020).

\subsection{Participantes do processo de gestão}

Os gestores e/ou coordenadores dos dispositivos precisam estar atentos para perceberem onde há necessidade de mudanças e melhorias, pois, ao estarem em uma posição de coordenação de todos os serviços prestados, necessitam ter uma 
visão e ouvidos ampliados a fim de não deixarem detalhes influenciadores importantes passarem despercebidos, onde poderão intervir de forma eficiente nas questões identificadas (Lima \& Guimarães, 2019).

As equipes multiprofissionais dos dispositivos também exercem papel de suma importância para o gerenciamento da rede, pois ao terem contato entre si e entre equipes de outros dispositivos elas têm a possibilidade de ver o funcionamento da rede e as demandas mais urgentes dos usuários, com isso podem entrar em conversação para a manutenção da rede como um todo (Schran et al. 2019 \& Barbosa, 2019).

Juntamente aos gestores e as equipes multiprofissionais, vêm os usuários do serviço, assumindo um grande papel para a gestão da rede, pois quem melhor para identificar as falhas na rede senão aquele que a usa? Com isso, eles têm grande participação para o processo através do dispositivo de reuniões, onde se reúnem com gestores e equipes para debaterem sobre a funcionalidade da rede como um todo (Silva; Lima \& Ruas, 2018).

É para o envolvimento dos usuários que o Conselho Nacional de Saúde juntamente com suas comissões intersetoriais existe, pois com seu caráter deliberativo e sendo composto, em sua maior parte por usuários, ele os dá a possibilidade de ter uma voz e de auxiliarem para que a rede funcione de forma eficiente e atenda a todas as suas demandas (Ministério da Saúde, 2016; Silva, Lima \& Ruas, 2018).

\section{Considerações Finais}

A Rede de Atenção Psicossocial é dotada de dispositivos dos três níveis de atenção, cada um tendo sua função no cuidado ao usuário, onde todos estão conectados entre si atuando paralelamente um ao outro, mas não de forma divergente. É necessário conhecimento aguçado por parte dos profissionais para que façam a rede ter funcionalidade efetiva.

A gestão da rede se dá pela articulação dos seus pontos de atenção, principalmente entre as equipes, utilizando os dispositivos que auxiliam nesse processo, a fim da garantia da prestação de serviços que atendam de forma objetiva e qualificada às demandas apresentadas.

Os gestores, profissionais e usuários são os responsáveis por fazer a gestão dessa rede como um todo de forma uniforme, onde devem entrar em consenso nas decisões, a fim de conseguirem suprir as necessidades e dar procedência no funcionamento dela sem que haja colapso e resolvendo o quanto de falhas puderem.

Ao analisar os artigos foi percebido a falta de estudos sobre a temática, devido a dificuldade de localização de pesquisas referente a mesma, e a não normalização do processo de trabalho em conjunto entre gestores, profissionais e usuários, visto que nem todos os estudos se voltavam aos três como peças fundamentais e intrínsecas uma à outra nesse processo de gestão da rede.

Com base nos estudos encontrados, foi percebido a necessidade de estudos futuros abordarem de forma mais detalhada e integral sobre o funcionamento da gestão da Rede de Atenção Psicossocial e os participantes dessa gestão. Sendo necessário também mais pesquisas acerca da temática, levando em consideração o número limitado de estudos encontrados.

\section{Referências}

Barbosa, T. L. (2019). Desafios na construção de uma Rede de Atenção Psicossocial no município de Manaus (AM): discursos e práticas de gestores e profissionais. 100 f. Tese (Doutorado) - Programa de Pós-Graduação em Saúde Coletiva, Universidade do Estado do Rio de Janeiro, Rio de Janeiro. http://www.bdtd.uerj.br/tde_busca/arquivo.php?codArquivo=16740.

Bardin, L. (2011). Análise de conteúdo. São Paulo: Edições 70.

Braga, F. S. et al. (2020). Meios de trabalho do enfermeiro na articulação da Rede de Atenção Psicossocial. Rev. Gaúcha Enferm. 41, 1-8. https://www.scielo.br/j/rgenf/a/Fk8DBtzF7VSpf8FN8LMJSGH/?lang=pt. 
Brasil. (2011). Portaria $n^{\circ} 3.088$, de 23 de dezembro de 2011. Institui a Rede de Atenção Psicossocial para pessoas com sofrimento ou transtorno mental e com necessidades decorrentes do uso de crack, álcool e outras drogas, no âmbito do Sistema Único de Saúde. Diário Oficial [da] República Federativa do Brasil. Brasília, DF; Ministério da Saúde. https://bvsms.saude.gov.br/bvs/saudelegis/gm/2011/prt3088_23_12_2011_rep.html.

Brasil.(2016). Conselho Nacional de Saúde. Conselho Nacional de Saúde. Brasília, DF; Ministério da Saúde. http://conselho.saude.gov.br/apresentacao/index.htm.

Brasil. (2018). Conselho Nacional de Saúde. Brasília, DF; Ministério da Saúde. http://conselho.saude.gov.br/apresentacao-cns.

Brasil.(2018). Comissão Intersetorial de Saúde Mental. Conselho Nacional de Saúde. Brasília, DF; Ministério da Saúde. https://conselho.saude.gov.br/Web_comissoes/cism/index.html.

Brasil. (2018). Comissão Intersetorial de Saúde Mental. Brasília, DF; Ministério da Saúde. http://conselho.saude.gov.br/contato-cns/84-comissoes/cism/796comissao-intersetorial-de-saude-mental.

Brasil. (2019). Saúde mental: o que é, doenças, tratamentos e direitos [online]. Brasília, DF; Ministério da Saúde. https://saude.gov.br/saude-de-a-z/saudemental.

Brasil. (2020). Nota pública: CNS defende Política Nacional de Saúde Mental desinstitucionalizadora, antimanicomial e com participação social. Brasília, DF; Ministério da Saúde. http://conselho.saude.gov.br/ultimas-noticias-cns/1505-nota-publica-cns-defende-politica-nacional-de-saude-mentaldesinstitucionalizadora-antimanicomial-e-com-participacao-social.

Cardoso, M. R. O. et al. (2021). O caminho trilhado por usuários de um Centro de Atenção Psicossocial do Estado do Pará: construindo itinerários na busca do cuidado. Mental, 11(20), 91-116. http://pepsic.bvsalud.org/pdf/mental/v11n20/v11n20a06.pdf.

Dimenstein, M. et al. (2021). A saúde mental e atenção psicossocial: regionalização e gestão do cuidado integral do SUS. Salud \& Sociedad, 9(1), 70-85. https://revistas.ucn.cl/index.php/saludysociedad/article/view/2869.

EndNote Web (2021). [place unknown]; https://access.clarivate.com/login?app=endnote

Hurso. (2020). OMS considera depressão uma epidemia global. Hospital Estadual de Urgências da Região Sudoeste Dr. Albanir Faleiros Machado. http://hursosantahelena.org.br/noticias/oms-considera-depressao-epidemia-global/.

Ibes. (2016). Afinal, qual é o papel do Conselho Nacional de Saúde? Grupo IBES. https://www.ibes.med.br/afinal-qual-e-o-papel-do-conselho-nacional-desaude/.

Leite, L. S., Rocha, K. B. \& Santos, L. M. (2017). A tessitura dos encontros da Rede de Atenção Psicossocial. Trab. Educ. Saúde, Rio de Janeiro, p. 1-18. https://www.researchgate.net/publication/321845657_A_TESSITURA_DOS_ENCONTROS_DA_REDE_DE_ATENCAO_PSICOSSOCIAL.

Lima, D. K. R. R. \& Guimarães, J. (2019). Articulação da Rede de Atenção Psicossocial e continuidade do cuidado em território: problematizando possíveis relações. Physis: Revista de Saúde Coletiva, Rio de Janeiro, 29(3), 1-20.https://www.scielosp.org/article/physis/2019.v29n3/e290310/pt/.

Mendes, K. D. S., Silveira, R. C. C. P. \& Galvão, C. M. (2008). Revisão integrativa: método de pesquisa para a incorporação de evidências na saúde e na enfermagem. Texto Contexto Enferm, Florianópolis, 17(4),758-764. http://www.scielo.br/pdf/tce/v17n4/18.pdf.

Nóbrega, M. P. S. S., Silva, G. B. F.\& Sena, A. C. R. (2016). Funcionamento da Rede de Atenção Psicossocial-RAPS no município de São Paulo, Brasil: perspectivas para o cuidado em Saúde Mental. Investigação Qualitativa em Saúde, 2, 41-49. https://proceedings.ciaiq.org/index.php/ciaiq2016/article/view/735/722.

Pessoa, K. L. V. et al. (2018). Gestão do cuidado e interdisciplinaridade: desafios do cotidiano da atenção psicossocial. Rev. Salud Pública, v. 20, n. 6, p. 692698. http://www.scielo.org.co/pdf/rsap/v20n6/0124-0064-rsap-20-06-692.pdf.

Peters, M. D. J. et al. (2015) Guidance for conducting systematic scoping reviews. Int J Evid Based Healthc ,13(3), 141146.https://www.researchgate.net/publication/319713049_2017_Guidance_for_the_Conduct_of_JBI_Scoping_Reviews?enrichId=rgreq-2c63bf47a03bf1 c379fed09bf9a175b4-XXX\&amp;enrichSource=Y292ZXJQYWdiOzMxOTcxMzA0OTtBUzo1NDA5MDcxMjY4ODY0MDBAMTU wNTk3MzcxNjg4MA\%3D\%3 D\&amp;el=1_x_2\&amp.

Quinderé, P. H. D., Jorge, M. S. B. \& Franco, T. B. (2021). Rede de Atenção Psicossocial: qual o lugar da saúde mental?. Physis: Revista de Saúde Coletiva. Rio de Janeiro, 24(1), 253-271. https://www.scielo.br/j/physis/a/7zsMFLdhyQZJF7GmtPBTj6s/abstract/?lang=pt

Schran, L. S. et al (2019). Percepção da equipe multidisciplinar sobre a estrutura dos serviços de saúde mental: estudo fenomenológico. Rev. Gaúcha Enferm., 40, 1-7. https://seer.ufrgs.br/RevistaGauchadeEnfermagem/article/view/88847/51182.

Silva, S. N., Lima, M. G. \& Ruas, C. M. (2018). Avaliação de Serviços de Saúde Mental Brasileiros: satisfação dos usuários e fatores associados. Ciênc. saúde colet.,23(11), 3799-3810. https://www.scielosp.org/article/csc/2018.v23n11/3799-3810/.

Silva, A. P. et al. (2021). Os desafios da organização em rede na atenção psicossocial especializada: o caso do Recife. Saúde debate, 128(45), 66-80. https://www.scielosp.org/article/sdeb/2021.v45n128/66-80/.

Zanardo, G. L. P., Bianchessi, D. L. C. \& Rocha, K. B. (2018). Dispositivos e conexões da Rede de Atenção Psicossocial (RAPS) de Porto Alegre - RS. Estudos Interdisciplinares em Psicologia, Londrina, 9(3), 80-101. https://repositorio.pucrs.br/dspace/bitstream/10923/16052/2/Dispositivos_e_ conexoes_da_Rede_de_Atencao_Psicossocial_RAPS_de_Porto_Alegre_RS.pdf. 